\title{
Fourth Refresher Course in Materials Preparation and Measurement of Properties
}

\author{
Sponsored by \\ Indian Academy of Sciences, Bangalore, \\ Indian National Science Academy, New Delhi \\ The National Academy of Sciences, Allahabad, India
}

The fourth Refresher Course in Materials Preparation and Measurement of Properties will be held from 10-25 February 2015 at the premises of the Indian Academy of Sciences in Jalahalli, Bangalore. This Course will provide hands-on experience in thin film preparation by evaporation and sputtering, making pellets of materials and attaching contacts, measurement of thermal conductivity, thermal diffusivity, electrical resistivity of alloys of metals, thermoelectric property, dielectric, piezoelectric and ferroelectric properties, magnetic properties, superconductivity, phase transitions, and differential thermal analysis. The Course will be jointly directed by $\mathrm{T}$ G Ramesh and R Srinivasan.

Refresher courses in Experimental Physics conducted by R Srinivasan have been very successful. Total number of seats in the Course will be about 25 .

The Course is essentially meant for teachers in universities and colleges, teaching Physics or Materials Science. A few students studying MSc-I Physics or Materials Science courses will also be admitted. It has been brought to our attention that teachers in universities/colleges who attend Refresher Courses of two-week duration are also entitled to be considered for promotion.

Interested applicants must submit their application ONLINE by clicking on the following link http://web-japps.ias.ac.in:8080/Refreshcourse/RCMP4.jsp

In the case of teacher applicants, a hard copy of the application should be forwarded by the applicant through the Head of the Institution to the address given below, stating that leave will be sanctioned if the applicant is selected for the Course.

The Coordinator, Science Education Programme, Indian Academy of Sciences, C V Raman Avenue, Sadashivanagar, Bangalore 560080.

For any further query, please Email to sep@ias.ernet.in. Selected participants will be intimated by Email around 20 December 2014. They will be paid the actual to and fro train fare not exceeding three-tier AC fare. Boarding and lodging in Bangalore will be taken care of by the Academy.

Last date for receiving applications will be 15 December 2014. 